\title{
Le parasitisme des poissons Siluroidei : un danger pour l'aquaculture ?
}

\author{
I ouis Euzet (1) et Antoine Pariselle (2)
}

(1) Station méditerranéenne de l'Environnement littoral, 1, quai de la Daurade, 34200 Sële, France.

(2) ORSTOM, Groupe aquaculture continentale méditerranéenne et tropicale (GAMET), BP 5095, 34032 Montpellier Cedex 1, France. E-mail: parisel]@orstom.rio.net

Accepté le 31 juillet 1996.

Euzet L., A. Pariselle. In: The biology and culture of catfishes. M. Legendre, J.-P. Proteau eds. Aquat. Living Resour., 1996, Vol. 9, Hors série, 145-151

Parasitism of catfishes: a danger for aquaculture?

\begin{abstract}
All the known groups of parasites, both protozoan and metazoan, have been recorded from Siluroidei. By their location on the host one can distinguish: ectoparasites, living on the tegument and/or the gills; mesoparasites, found mainly in the digestive tract and endoparasites, located in the body (cavities or tissues). Three factors seem to be important to determine parasite accumulations: 1) the multiplication rate, linked to the reproductive mode, particularly asexual; 2) the size of parasitic organism, which can facilitate the host invasion and 3 ) the life cycle, direct (monoxenous or holoxenous) or indirect (heteroxenous). The examination of papers related to Siluroidei showed that the most studied type of pathogenic agents or parasites depend on the state of knowledge in the country where farming is practised. The most currently quoted parasitic diseases on Ictalurus punctatus are detailed. Parasitism on other Siluroidei of actual or potential importance for aquaculture is discussed.
\end{abstract}

Keywords: Parasites, pathology, Siluroidei, fish culture.

Résumé

Tous les grands groupes de parasites de poissons, aussi bien Protozoaires que Métazoaires, ont été signalés chez les Siluroídei. D'après leur localisation, chez l'hôte on peut distinguer: les ectoparasites qui vivent sur le tégument et/ou sur les branchies; les mésoparasites que l'on rencontre surtout dans le système digestif; les endoparasites qui sont dans le corps (cavités ou tissus). Une accumulation anormale de parasites, par rapport à l'équilibre généralement observé dans la nature, engendre des effets pathogènes chez les poissons en aquaculture. Trois facteurs semblent importants dans le déterminisme de cette accumulation: 1) la vitesse de multiplication, liée au mode de reproduction, en particulier asexué ; 2) la taille de l'organisme parasite, qui peut faciliter l'envahissement de l'hôte et 3) le type de cycle biologique, direct (monoxène ou holoxène), ou indirect (hétéroxène). La bibliographie consacrée aux Siluroidei montre que les agents pathogènes ou les parasites les plus étudiés dépendent de la forme d'élevage pratiquée (industrielle ou artisanale). Les parasitoses les plus couramment citées pour leur importance chez Ictalurus punctatus sont détaillées. La question du parasitisme des autres Siluroidei élevés ou potentiellement intéressants est posée.

Mots-clés : Parasites, pathologie, Siluroidei, aquaculture. 


\section{INTRODUCTION}

De nombreux parasites sont actuellement connus chez les Siluroidei et on en découvre sans cesse de nouveaux. Pourquoi et comment ces parasites peuventils présenter un danger pour l'aquaculture?

En général et chez les poisson en particulier l'effet pathogène des parasites, dans les conditions naturelles, est réduit, consćquence de l'équilibre établi au cours de l'évolution dans le système hôtc/parasite. En aquaculture c'est l'accumulation, chez un individu hôte, d'un organisme étranger: virus, bactérie, ou parasite, qui entraîne un effet pathogène dont la gravité est proportionnclle à cette accumulation (Combes, 1990). La présence d'un métazoaire parasite peut favoriser la multiplication de protistes potentiellement pathogènes, un tel phénomène d'interdépendance a été décrit par Benajiba et al. (1995) chez. Anguilla anguilla. Les cas où un petit nombre de métazoaires parasites sont responsables de la mort de leur hôte sont extrêmement rares et dépendent principalement de leur localisation (encéphale par exemple) ou sont liés à la taille réduite de l'hôte (alevins).

D'après Euzct (1990) on peut distinguer, d'après la position qu'ils occupent chez l'hôtc, trois types de parasites.

- Les ectoparasites vivent sur le corps ou les branchies du poisson hôte et sont donc en contact direct avec le milieu extérieur. Les slades de dispersion, destinés à parasiter un nouvel hôte, n'ont donc aucune pcinc à atteindre ce milieu.

- Les mésoparasites sont dans l'hôte mais dans une cavité possédant une ouverture naturelle sur le milieu extérieur (pas exemple le tube digestif ou la vessie urinaire). Dans ce cas également les stades de dispersion peuvent atteindre facilement le milicu extérieur, mais pour le parasite cela implique un changement de milieu.

- Les endoparasites vivent à l'intérieur de l'hôte, soit dans les tissus (conjonctif par exemple), soit dans des cavités fermées (système circulatoire). Cela entraîne pour le parasitc l'obligation d'une effraction pour entrer el sortir de l'hôte. Lors de la sortic, comme dans le cas précédent, les stades de dispersion changent de milicu. Certaines formes peuvent avoir une multiplication asexuée, les individus formés persistant à l'intérieur de l'hôte, on parle alors d'un recrutement endogène (endogeneous agglomeration) (Dogiel, 1962).

\section{ACCUMULATION ET PATHOGÉNICITÉ}

Trois factcurs paraissent jouer un rôle majeur dans l'apparition d'une pathogénicité par accumulation.

\section{La vitesse de multiplication}

Chez un hôte, plus un parasite se multiplie activement, plus son accumulation peut être rapide et l'effet pathogène précoce. D'après une estimation grossière de leur vitesse de multiplication, on peut établir une série décroissante des organismes allant des crustacés aux virus, en passant par les helminthes, les protozoaires et les bactéries. Cette série peut être mise en parallèle avec l'importance estimée de la pathologie dont ces parasites sont responsables.

Il faut remarquer le rôle prépondérant des phénomèncs de multiplication végétative ou asexuée sur la vitesse de reproduction de ces organismes. Ainsi parmi les Monogènes ce sont les espèces du genre Gyrodactylus connues pour leur polyembryonie (un seul oeuf donnant à chaque fois quatre individus), qui sont le plus souvent reconnus comme responsables de mortalité par accumulation chez les poissons en élevage, Cyprinidae, Characidae (Paperna, 1982) et Siluroidci (Szidat, 1973), (Obiekezie et Taege, 1991).

\section{I a taille}

La taille de l'organisme étranger représente un autre facteur important. En effet, les virus et les bactéries, de taille extrêmement réduite, paraissent se jouer des membranes cellulaires, qu'ils peuvent traverser dans les deux sens. Ces organismes parviennent même à franchir des barrières plus conséquentes, comme la membrane basale des épithéliums. Cette possibilité existc aussi chez quelques protozoaires de petite taille, mais elle est limitée aux stades infestants, comme le sporoplasme des Microsporidies ou des Myxosporidies. Du fait de leur taille, la pénétration traumatique des métazoaires est plus rare. Lorsqu'elle existe la localisation du parasite est tissulaire, par exemple la métacercaire des Clinostomum (Trematoda) chez certains Siluroidei (Douëllou et Erlwanger, 1993).

\section{Le type de cycle biologique}

En fonction du nombre d'hôtes caractérisant le cycle biologique on distingue les parasites holo- et hétéroxènes.

- Les parasites holoxènes (ou monoxènes) ont un cycle direct avec un seul hôte. Le parasite circule d'un individu hôte à un autre individu hôte. Le stade de dispersion, qui est aussi le stade infestant, assure le passage dans le milieu extérieur.

- Les parasites hétéroxènes (cycle indirect) ont, entre un individu hôte définitif et un autre individu hôte définitif, un ou plusieurs hôtes intermédiaires où le parasite s'isole et se développe jusqu'au stade infestant. L'infestation de l'hôte définitif est généralement lié à son régime alimentaire.

Les ectoparasites ont, dans leur ensemble, un cycle holoxène. Le cycle des mésoparasites est soit holoxène, soit hétéroxène. Les endoparasites ont un cycle hétéroxène, mais l'hôte intermédiaire est souvent 
lui-même un ectoparasite, alors qualifié de vecteur. C'cst lui, en effet, qui après effraction, prend le parasite chez un individu et l'injecte ensuite, après transformation ou non, à un autre individu.

Dans les élevages, on a signalé de nombreux cas de pathologie, principalement dus à des ectoparasites holoxènes: Protozuaires (Kabata, 1985), Monogènes (Paperna, 1963; Silan et Maillard, 1986), Copépodes (Fryer, 1961). En effet, les formes de dispersion (kystes, cufs ou larves) libérées restent dans le bac d'élevage, elles n'ont aucune peine à trouver un nouvel hôtc. Chez les poissons l'accumulation du parasite prend alors une allure asymptotique conduisant à la pathogénie et à la mortalité.

Les méso- et les endoparasites hétéroxènes sont « théoriquement » moins dangereux car il est relativement facile de rompre le cycle biologique en éliminant un des hôtes intermédiaires lorsque celui-ci apparaît dans le milieu d'élevage. En réalité, ceci est plus délicat lorsqu'il s'agit d'un petit ectoparasite vecteur.

Cependant pour les endoparasites qui se sont installés après avoir sümonté les réactions de l'hôte, l'accumulation est facilitée par un recrutement endogène, les nombreux individus formés restant enfermés dans l'hôte. Dans ce cas on peut citer les virus, les bactéries et certains protozoaires.

\section{LES MALADIES PARASITAIRES CHEZ LES SILUROIDEI}

$\mathrm{Si}$ on dresse un bilan de la littérature consacrée au parasitisme des poissons Siluroidei, on note que, parmi les publications recensées entre 1974 et 1994, $57 \%$ portent sur l'étude de maladies infectieuses dues à des virus ou à des bactéries, et $43 \%$ sur l'étude de parasites (17\% pour les protozoaires, $26 \%$ pour les métazoaires) mais dont l'action pathogène n'est pas toujours évoquée.

$\mathrm{Si}$ on s'intéresse aux seuls Siluroidei faisant l'objet d'élevage, il faut remarquer le poids pris par les travaux consacrés à Ictalurus punctatus, qui représentent $50 \%$ de l'ensemble. Parmi ces travaux $78 \%$ portent sur les maladies infectieuses et $22 \%$ sur les maladies parasitaires ( $16 \%$ pour les protozoaires, $6 \%$ pour les métazoaires). Ces proportions sont comparables à celles relevées par MacMillan (1985) sur Ictalurus punctatus, où sur 68 publications traitant de la pathologie, $84 \%$ sont consacrées aux maladies infectieuses et $16 \%$ aux maladies parasitaires.

Chez les autres Siluroidei élevés, seulement $35 \%$ des publications portent sur la pathologie des maladies infectieuses et $65 \%$ sur les parasites (19\% protozoaires et $46 \%$ métazoaires), dans ce dernier cas il s'agit principalement de descriptions d'espèces nouvelles et exceptionnellement de pathologies.

Deux raisons peuvent expliquer ces différences (fig. 1), à la fois dans le nombre de publications et dans la proportion relative des agents pathogènes

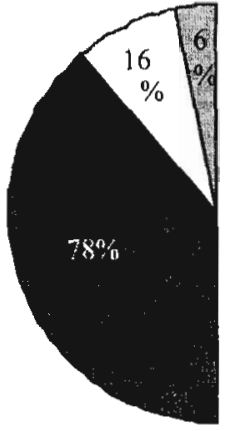

Ictalurus punctatus

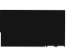

Maladies infectieuses

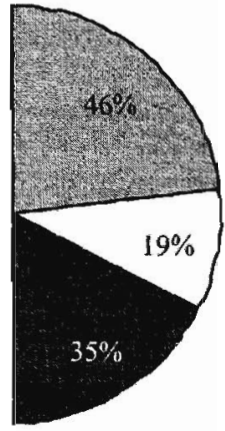

Autres Siluroidei élévés
Figure 1. - Proportions relatives des publications consacrécs aux maladies infectieuses, aux parasites protozoaires et métazoaires chez les Siluroidei faisunt l'objet d'élevage.

Percentage of references related to infectious diseases, protozoan and metazon parasites in Siluroidei important for aquaculture.

éludiés. La première est l'importance en Amérique du Nord des élevages du seul Ictalurus punctatus (200000 t/an) par rapport à la production, estimée à $150000 \mathrm{t} / \mathrm{an}$, pour tous les autres Siluroidei élevés (Silurus, Pangasius, Clarias, etc.); si ce dernier chiffre peut être contesté, il existe cependant une nette différence entre l'importance des élevages industriels d'Ictalurus et celle des élevages, souvent encore artisanaux, des autres Siluroidei. La seconde est l'importance des recherches très actives sur les maladies d'Ictalurus par rapport à la rareté des données sur la pathologie des Siluroidei dans le reste du monde.

Chez les Siluroidei, on a signalé tous les principaux groupes de parasites de poissons, aussi bien protozoaires (Lom et Dykovà, 1992) que métazoaires (Khalil, 1971; Kabata, 1985 ; Paperna, 1982; etc.). Ainsi Fagbenro et al. (1993) signalent chez Heterobranchus bidorsalis 23 genres différents de parasites. La richesse parasitaire des représentants des genres Clarias, Chrysichthys, Ictalurus et Silurus, poissons qui font l'objet d'élevage, est assez bien connue. Pour tous les autres Siluroidei, Pangasius en particulier, les données restent fragmentaires.

Pour Ictalurus punctatus, nous avons relevé (tableau 1) 44 espèces de parasites toutes potentiellement pathogènes (Hoffman, 1967). Plusieurs Protozoaires (Henneguya, Ambiphrya, Chilodonella, Trichodina) ainsi qu'un Monogène (Gyrodactylus) ont effectivement entraîné à plusieurs reprises des cas de grave pathologie (Bowser et Conroy, 1985; MacMillan, 1985). Deux protozoaires, qualifiés d'opportunistes car ils s'attaquent souvent à des poissons affaiblis en particulier par d'autres parasites, le flagellé Ichthyobodo necator et le 
Tableau 1. - Liste des parasites d'/ctalurus punctatus.

List of Ictalurus punctatus parasites.

\section{PROTOZOAIRES}

Mastigophora

khthysbodo necator

Myxozoa

Henneguya adiposa

Henneguya diversis.

Henneguya exilis

Henneguya limatula

Henneguya longicauda

Henneguya postexilis

Myxidium bellum

Ciliophora

Ichthyophthirius multifilis

Ambiphrya ameiuri

Amphileptus voracus

Capriania pisciam

Chilodonella sp.

Epistilys sp.

Trichophrya sp.

Trichodina discoidea

Trichodina perforata

Trichodina vallata

Trichodinella symmetrica

\section{METAZOAIRES}

Monogenea

Ligirtaluridus floridanus

Ligictaluridus pricei

Gyrodactylus ictaluri
Trematoda

Acendextra aniuri

Alloglossidium corti

Azrgia angusticanda

Crepidostomum cornutum

Macroderoides sp.

Megalogenia icialuri

Microphallus opacus

Parastiotrema oflawanensis

Phyllodisomum lacustri

Vietosoma parvam

Apophallus tenastus (metacercaria)

Diplostomulum (metacercaria)

Cestoda

Corollobothrium fimhriatum

Haplohothrium globuliforme

Megahylacoides giganteum

Nematoda

Camallanus oncephalus

Spinitectus gracilis

Acanthocephala

Leptorhynchoides thecatus

Pomphorhynchus sp.

Hirudinoidea

Myzobdella moorei

Copepoda

Actheres pimelodi

Ergasilus versicolor cilié Ichthyophthirius multifilis, ont aussi causé des mortalités importantes dans les élevages. Ichthyobodo, ectoparasite de nombreux Téléostéens dulçaquicoles, fait surtout des victimes chez les alevins, les juvéniles et les adultes affaiblis de Siluroidei (MacMillan, 1985). Ce petit flagellé $(10$ à $20 \mu \mathrm{m})$ enfonce deux de ses flagelles dans une cellule de l'épithélium tégumentaire ou branchial (Smith et Inslee, 1980). Une fois fixé, il se nourrit au dépens de la cellule qui est finalement détruite. Ichthyobodo se multiplie par division binaire, les individus formés se détachent alors, nagent grâce à leurs flagelles et vont se piquer dans de nouvelles cellules épithéliales qui sont à leur tour détruites. En élevage, l'invasion est très rapide et la mortalité élevée. Les sécrétions muqueuses, que l'on peut considérer comme une tentative de défense du poisson, sont abondantes et, au niveau des branchies, réduisent fortement les échanges respiratoires. Le seul moyen de diagnostique de cette parasitose est l'examen, au microscope, d'un frottis de l'épithélium cutané ou branchial.

Le cilié, Ichthyophthirius multifilis, est responsable de la maladie dite des points blancs. Les jeunes y sont particulièrement sensibles. Le cycle holoxène peut ĉtre résumé de la manière suivante: la forme infestantc (tomite) est un petit cilié piriforme de $50 \mu \mathrm{m}$ dc long avec une région apicale non ciliée. Cette région permet au parasite de pénétrer dans l'épithélium du poisson. Il forme alors des galeries dans l'épiderme et le derme où il commence à se nourrir et se multiplier (Ewing et al., 1988). Il semble que la conjugaison (type particulier de reproduction sexuée) se déroule pendant cctte phase. Les individus résultants (trophozoïte) grossissent rapidement (jusqu'à $1 \mathrm{~mm}$ de diamètre). L'épiderme qui les surmonte s'amincit et se rompt. Le protozoaire libéré tombe sur le fond et s'entoure d'une enveloppe kystique. A l'intérieur du kyste une multiplication asexuée conduit à la formation de 1000 à 2000 tomites (le nombre dépend de la taille du trophozoïte qui a quitté l'hôte). Les tomites une fois libérés nagent grâce à leur couverture ciliée et vont assurer de nouvelles infestations. Ce parasite peut se révéler particulièrement dangereux puisque progressivement les kystes formés restant dans les bacs d'élevage libèrent dans le milieu de plus en plus de tomites infestants. En outre, le cycle complet, de 
tomite à tomite, est très rapide: quatre jours seulement à $25^{\circ} \mathrm{C}$.

Ichthyophthirius, ainsi que Ichthyobodo, qui parasitent de nombreux Téléostéens, sont aussi dangereux pour les élevages en cage puisque les formes infestantes peuvent provenir de poissons sauvages infestés natureliement (Guevara Pozo et Lopez Román, 1969).

Un parasitisme, avec effets pathogènes, a été signalé chez d'autres Siluroidei. Par exemple les Myxosporidies Henneguya sp. chez Ictalurus punctatus (Meyer, 1969), Henneguya chrysichthyi sur les branchies de Chrysichthys nigrodigitatus (Obiekezie et al., 1992) ou Henneguya camerounensis chez Synodontis batesii (Fomena et Bouix, 1987). Les kystes de ces Myxozoaires peuvent, dans les cas d'infestations sévères, recouvrir les branchies, entraînant des perturbations respiratoires et d'importantes hémorragies. De même qu'avec Gyrodactylus ictaluri (Monogène) chez Ictalurus punctatus (Cone et Odense, 1984) on a relevé des cas de pathologies parasitaires chez Clarias gariepinus avec Gyrodactylus groshafti (Obiekerie et Taege, 1991), L'accumulation du Monogène Ancylodiscoides vistulensis, dont la reproduction ne comporte cependant pas de polycmbryonie, s'est avérée pathogène dans les élevages de Silurus glanis (Szekeli et Molnar, 1990). Chez Clarias batrachus les Monogènes comme Quadriacanthus kobiensis sont donnés comme les pathogènes les plus fréquents dans les écloseries en Indonésie (Supriyadi et al., 1986, Komarudin et al., 1991 a,b). L'accumulation de ces parasites est le facteur principal de mortalité, ainsi $300 G$. groshafti suffisent à tuer un alevin de Clarias de taille inférieure à $30 \mathrm{~mm}$ (Obickezie et Taege, 1991).

Les exemples précédents ont été pris parmi les ectoparasites, mais on connaît aussi des méso- et des endoparasites potentiellement dangereux pour les Siluroidei. Ainsi le Cestode Polyonchobothrium clarias, parasite intestinal de Clarias mossambicus (syn. C. gariepinus), est cité par Wabuke-Bunoti (1980) comme affectant sérieusement le fonctionnement du foie de ce poisson.

Parmi les parasites potentiellement pathogènes Davronov (1987) a signalé chez Silurus glanis la présence d'une coccidie Eimeria siluri. Le cycle de ce sporozoaire, qui se déroule en partie dans les cellules de l'épithélium intestinal du poisson (mésoparasite), est comparable à celui des Eimeria connues chez les oiseaux et les mammiferes. Chez Silurus glanis, un autre sporozoaire Hemogregarina turkestiana a été observé dans le sang. Ce protozoaire, endoparasite à cycle hétéroxène, existe en général dans la nature sous une forme chronique avec de faibles intensités et on peut craindre la contamination des élevages en bassins et surtout en cages, si dans le milieu existe la sangsue vectrice. L'accumulation devient alors pratiquement inévitable et la pathologie peut apparaître selon un processus comparable à celui des Plasmodium responsables du paludisme chez l'homme.
Pour l'aquaculture des Siluroidei, il semble que les Crustacés Argulidae, dont on connaît de nombreuses espèces chez les poissons africains (Van As et Basson, 1988), représentent un réel danger. Pour atteindre le système circulatoire, ces crustacés cctoparasites hématophages, percent la peau du poisson. Lors d'accumulations, ils affaiblissent les poissons-hôtes et les plaies cutanées, résultant de l'action des pièces buccales, sont la voie ouverte aux organismes infecticux. Les Argulidae, capables de se déplacer d'un hôte à l'autre, pourraient ainsi jouer le rôle de vecteur dans le cycle d'endoparasites.

On pourrait multiplier les exemples comme le cas de l'action traumatisante des cercaires de Clinostomum lors de leur pénétration et de leur enkystement en métacercaires dans les tissus du Siluroidei hôte intcrmédiaire de ce Trématode d'oiseau (Douëllou et Erlwanger, 1993).

\section{DISCUSSION}

Le développement de l'aquaculture des Siluroidei doit tenir compte de quatre phénomènes liés à la pathologie parasitaire, et malheureusement trop souvent négligés.

\section{Estimation de la pathologie parasitaire en milieu naturel}

Les Siluroidei sauvages sont toujours porteurs de nombreux parasitcs. Ainsi en Côte d'Ivoire nous avons observé, chez Clarias gariepinus, cinq espèces différentes de Quandriacanthus (Monogenea) sur les branchies, de nombreux Polyonchobothrium clarias (Cestoda) fixés à la paroi intestinale et des larves de Contracaecum (Nematoda) encapsulées dans le mésentère et la paroi abdominale.

Dans la nature il existe un équilibre entre l'action du parasite et les réactions de l'hôte. Cependant chez les alevins et les juvéniles, où les défenses « immunitaires » ne sont pas encore en place, l'action des germes infectieux et des parasites entraîne des mortalités sans que l'on puisse en déceler les causes et l'importance. Du fait de leur petite taille, ces alevins disparaissent rapidement après leur mort. C'est pourquoi il est probable que l'impact du parasitisme sur la dynamique des populations de poissons est toujours sous-estimé.

\section{Estimation de la pathologie parasitaire en aquaculture}

En élevage, la mortalité due aux parasites est souvent mal prise en compte car elle revêt une forme discrète sans rapport avec les mortalités massives et rapides dues à l'action des maladies infectieuses.

Dans les élevages intensifs où la densité des hôtes est élevée, la majorité des parasites (virus et bactéries compris) ne se répartissent pas de manière régulière 
entre les individus-hôtes, mais souvent selon une répartition agrégative ou surdispersion (Langlais et Silan, 1995). Dans une telle population, un petit nombre d'individus hyperparasités sont la source des stades infestants qui vont s'installer sur les autres individus, cependant moins parasités. L'accumulation continue entraine la mort des individus hyperinfestés. Parmi la population restante, certains individus, peutêtre les plus affaiblis, vont accumuler à leur tour les parasites, devenir hyperinfestés et disparaître. Lors des traitements anti-parasitaires ce sont les individus hyperinfestés qui supportent mal le trailement et meurent. Le parasitisme paraît endigué, car on ne décèle plus de mortalité, mais plus ou moins rapidement le phénomène vá se reproduire avec apparition et mort d'individus hyperinfestés (Silan et Maillard, 1986). Avec le temps le phénomène se ralentit, à mesure qu'on se rapproche des conditions naturelles, c'est-à-dire des charges au mètrc cube qui ne sont plus économiquement intéressantes pour l'aquaculture.

\section{Introductions d'espèces}

L'introduction, sans contrôle sanitairc rigoureux, d'espèces dans une nouvelle biocénose représente un risque majeur à la fois pour l'espèce introduite mais aussi pour les espèces autochtones. La mise en contact de ces deux types de poissons peut entraîner, par transferts latéraux de parasites, une rupture de l'équilibre naturel hôtc/parasite. Cette rupture induit souvent une pathologie grave, par accumulation rapide des parasites transfuges, aussi bien chez les poissons introduits que chez les autochtones (Combes, 1995).

\section{Le parasitisme des hybrides}

En aquaculture on a de plus en plus tendance à réaliser des hybridations inter-spécifiques afin d'améljorer les performancés des poissons mis en élevage. Outre les possibilités de transferts latéraux les parasites peuvent présenter un nouveau type de danger. Obiekezie (1991) signale ainsi une infestation importante d'alevins des hybrides de Clarias gariepinus X Heterobranchus bidorsalis par une Myxosporidie du genre Henneguya alors que dans la même écloserie les alevins des espèces parentales sont indemnes de ce parasitc. Plusieurs cspèces d'Henneguya sont connues comme étant hautement pathogènes chez les Siluroidei.

\section{CONCLUSION}

Dans tous les cas, les modifications des conditions naturelles (spatiales et populationelles) résultant de la mise en ćlevage, perturbant ainsi l'équilibre hôte-parasite, créent un environnement favorable aux épirooties. C'est pourquoi, en fonction des phénomènes dont nous venons de souligner l'importance, il nous paraît indispensable, avant toute tentative d'élevage, de réaliser dans le milieu naturel une ćtude approfondie, taxonomique et biologique, des parasites pathogènes potentiels. Cette recherche devrait permettre de déterminer, en fonction des parasites reconnus, les prophylaxics à prévoir lors de la mise en élevage.

\section{RÉFÉRENCES}

Benajiba M.H., P. Silan, A. Marquies, G. Bouix 1995. Protozoaires et métazoaires parasites de l'anguille Anguilla anguilla: interdépendance des populations parasitaires et coexistence de parasites potentiels. $A n n$. Sci. Nat., Zool. Paris 16, 1-6.

Bowser P.R., J.D. Conroy 1985. Histopathology of gill lesions in channel cat fish associated with Henneguya. J. Widl. Dis. 21, 177-179.

Combes C. 1990 . When is a parasite pathogen? Metazoan parasitic diseases: concluding remarks. In: Pathology in marine science. Academic Press Inc. Publisher, 331-340).

Combes C. 1995. Interactions durables. F́cologic et évoJution du parasitisme. Coll. Ecologie 26, Masson ed. Paris, 524 p.

Cone D.K., P.H. Odense 1984. Pathology of five species of Gyrodactylus Nordmann, 1832 (Monngened). Can. J. Zool. 62, 1084-1088.

Davronov O. 1987. Coccidia from fishes of Uzbekistan. Parazitologiya (Leningr.) 21, 115-120.

Dogiel V.A. 1962. General Parasitology (transl. Z. Kahata 1964). Oliver and Boyd Publishers, London, 516 p.

Douëllou L., K.H. Erlwanger 1993. Occurrence and distribution of two Clinostomatid metacercariac in fishes from Lake Kariba, Zimbabwe. Trans. Zimbabwe Sci. A.ssoc. 66, 35-40.

Euzet L. 1990. Ecologie et parasitologie. Bull. Ecol. 20 (1989), 277-280.

Ewing M.S., S.A. Ewing, K.M. Kocan 1988. Ichthyophthirius (Ciliophora): Population studies suggest rcproduction in host epithelium. Protozool. 35, 549-552.

Fagbenro O.A., C.O. Adedire, E.A. Owoseeni, E.O. Atoyunde 1993. Studies on the biology and aquaculture potential of feral catfish Heterobranchus bidorsalis (Genffroy Saint Hilaire, 1809) (Clariidae). Trop. Zool. 6, 67-79.

Fomena A., G. Bouix 1987. Contribution à l'étude des Myxosporidies des poissons d'eau douce du Cameroun. III. Espèces nouvelles du genre Henneguya Thelohan, 1892 et Thelohanellus Kudo, 1933. Rev. Zool. Afr. 101, 43-53.

Fryer G. 1961. The parasitic Copepoda and Branchiura of the Lake Victoria and the Victoria Nile. Proc. Zool. Suc. London 137, 41-60.

Guevara Pozo D., R. Lopez Román 1969. Ichyhyophtirius multifiliis Fouquet, 1876. Muy probable causa de la gran mortandad ocasionada en los barbos de algunos pantanos de la provincia da Granada. Rev. Iher. Parasitol. 29, 197-218.

Hoffman G.L. 1967. Parasites of North American Freshwater Fishes. University of California Press Publishers, Berkeley, 486 p. 
Kabata Z. 1985. Parasites and diseases of fish cultured in the tropics. Taylor, Francis Publishers, London, 318 p.

Khalil L. 1971. Check list of the Helminth parasites of African freshwater fishes. Commonwealth Institute of Helminthology. Tech. Comm. 42, CAB Publishers St. Albans, U.K. 80 p.

Komarudin O., M. Shariff, F. Shaharom 1991a. The effects of Quadriacanthus kobiensis Ha Ky, 1968 (Monogenea) on the growth of walking catfish Clarias batrachus L. Bull. Penel. Perik. Darat 3, 81-87.

Komarudin O., M. Shariff, F. Shaharom $1991 b$. Toxicity of sodium chloride to Clarias batrachus (Linnaeus) and its effectiveness as a chemotherapeutant for Quadriacanthus kobiensis Ha Ky, 1968 infections. Dis. Asian Aquac. 1, 425-430.

Langlais M., P. Silan 1995. Theoretical and mathematical approach of some regulation mechanisms in a marine host-parasite system. J. Biol. Syst. 3, 559-568.

Lom J., E. Dykovà 1992. Protozoan parasites of fishes. Development in Aquaculture and fisheries sciences 26, Elsevier Publishers, Amsterdam, 315 p.

MacMillan J.R. 1985. Infectious diseases. In: Channel catfish culture. C.S. Tucker ed. Development in Aquaculture and Fisheries Sciences 15, Elsevier Publishers, Amsterdam, 405-496.

Meyer F.P. 1969. Henneguya infcctions. In: Progress Sport Fishery Research. Bur. Sport Fish. Wildl. Resour. Publ. $88,60$.

Obiekezie A.I. 1991. The principal pathogens and diseases of cultured fishes in Nigeria. Aquaculture in Africa. International Foundation for Science Publisher, Stockholm, 197-207.

Obiekezie A.I., H. Möller, K. Anders 1992. Diseases of the African estuarine catfish, Chrysichthys nigrodigitatus (Lacépède) from Cross River Estuary, Nigeria. J. Fish Biol. 32, 207-221.

Obiekezie A.I., M. Taege 1991. Mortalities in hatcheryreared fry of the African catfish, Clarias gariepinus
(Burchell) caused by Gyrodactylus groschafti Ergens, 1973. Bull. Eur. Assoc. Fish Pathol. 11, 82-85.

Paperna I. 1963. Dynamics of Dactylogyrus vastator Nybelin (Monogenea) Populations on the gills of carp fry in fish ponds. Bamidgeh, Bull. Fish Culture Israel 15, 31-50.

Paperna I. 1982. Parasites, infections et maladies du poisson en Afrique. CPCA, Doc. Tech. 7, 202 p.

Silan P., C. Maillard 1986. Modalités de l'infestation par Diplectanum aequans, monogène ectoparasite de Dicentrarchus labrax, en aquiculture. Éléments d'épidémiologie et de prophylaxie. In: Pathology in marine aquaculture C.P. Vivarès, J.-R. Bonami, E. Jaspers eds. Eur. Aquac. Soc. Publ. Bredene Belgium, Spec. Publ. 9, 139-152.

Smith C.E., T. Inslee 1980. Interlamellar Henneguya infestation in adult channel catfish lctalurus punctatus (Rafinesque). J. Fish Dis. 3, 257-260.

Supriyadi H.O., O. Komarudin, B. Yulianto 1986. Some parasites from fingcrling of walking catfish Clarias batrachus from Djakarti and Bogor, West Java. Bull. Penel. Perik. Darat 5, 59-61 (in Indonesian).

Szekeli C., K. Molnar 1990. Treatment of Ancylodiscoides vistulensis monogenean infestations of the European catfish (Silurus glanis). Bull. Europ. Assoc. Fish Pathol. 10, 74-77.

Szidat L. 1973. Morphologie und Verhalten von Paragyrodactylus superbus n. g., n. sp. erreger eines Fischerbens in Argentina. Ang. Parasitol. 14, 1-10.

Van As J.G., L. Basson 1988. Parasites of Sharptooth Catfish and their possible implication in aquaculture. In: The culture of Sharptonth Catfish, Clarias gariepinus, in Southern Africa. T. Hecht, W. Vys, P.J. Britz eds. South African National Scientific Progr. Rep. 153, 98-109.

Wabuke-Bunoti M.A.N. 1980. The prevalence and pathology of the Cestode Polyonchobothrium clarias (Woodland, 1925) in the teleost, Clarias mossambicus (Peters). J. Fish Dis. 3, 223-230. 